\title{
Supersonic plasma beams with controlled speed generated by the alternative low power hybrid ion engine (ALPHIE) for space propulsion
}

\author{
L. Conde, ${ }^{1, a)}$ J. L. Domenech-Garret, ${ }^{1}$ J. M. Donoso, ${ }^{1}$ J. Damba, ${ }^{1}$ S. P. Tierno, ${ }^{1, b)}$ \\ E. Alamillo-Gamboa, ${ }^{1}$ and M. A. Castillo ${ }^{2}$ \\ ${ }^{1}$ Departamento de Física Aplicada, ETS Ingeniería Aeronáutica y del Espacio, Universidad Politécnica de \\ Madrid, 28040 Madrid, Spain \\ ${ }^{2}$ Aernnova Aerospace SAU, Avenida de Manoteras 20, 28050 Madrid, Spain
}

(Received 20 September 2017; accepted 5 December 2017; published online 27 December 2017)

\begin{abstract}
The characteristics of supersonic ion beams from the alternative low power hybrid ion engine (ALPHIE) are discussed. This simple concept of a DC powered plasma accelerator that only needs one electron source for both neutral gas ionization and ion beam neutralization is also examined. The plasma production and space charge neutralization processes are thus coupled in this plasma thruster that has a total DC power consumption of below $450 \mathrm{~W}$, and uses xenon or argon gas as a propellant. The operation parameters of the plasma engine are studied in the laboratory in connection with the ion energy distribution function obtained with a retarding-field energy analyzer. The ALPHIE plasma beam expansion produces a mesothermal plasma flow with two-peaked ion energy distribution functions composed of low and high speed ion groups. The characteristic drift velocities of the fast ion groups, in the range $36.6-43.5 \mathrm{Km} / \mathrm{s}$, are controlled by the acceleration voltage. These supersonic speeds are higher than the typical ion sound velocities of the low energy ion group produced by the expansion of the plasma jet. The temperatures of the slow ion population lead to ion Debye lengths longer than the electron Debye lengths. Furthermore, the electron impact ionization can coexist with collisional ionization by fast ions downstream the grids. Finally, the performance characteristics and comparisons with other plasma accelerator schemes are also discussed. Published by AIP Publishing. https://doi.org/10.1063/1.5005881
\end{abstract}

\section{INTRODUCTION}

Plasma engines for space propulsion produce streams of high-velocity ions. Thrust is imparted to the spacecraft by the plasma jet created when electrons are added to this fast ion beam to neutralize the space charge effects. ${ }^{1-6}$

Electric propulsion (EP) systems accelerate ions to exhaust speeds $v_{e x}$ one or two orders of magnitude higher than classical chemical engines, leading to a larger specific impulse $I_{s p}$. This characteristic drastically reduces the amount of propellant, allowing for longer mission times and heavier payloads. ${ }^{1}$ The disadvantage is the low mass ion production rate $\dot{m}_{i}$ which is constrained by reduced electric power that can be made available on board, subsequently limiting the achievable thrust levels. ${ }^{1-5}$

EP systems are characterized by low thrusts $T=\dot{m}_{i} v_{e x}$ and high specific impulses. ${ }^{1-5}$ These features are essential for orbital maneuvers, station keeping, flight formation and/ or end-of-life disposal of small satellites $(50-250 \mathrm{Kg})$ of constellations intended for global internet coverage. They roughly require thrust levels in the range $0.1-10 \mathrm{mN}$ and electric power below $500 \mathrm{~W}$.

Here, we are specifically concerned with EP systems for these small and medium-sized satellites that are DC powered and operated in a steady state. This consideration excludes microwave-based engines such as the Radio Frequency Ion

\footnotetext{
${ }^{\text {a) Electronic mail: luis.conde@upm.es }}$

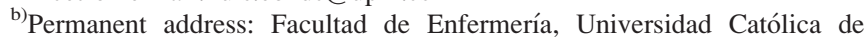
Valencia, 46007 Valencia, Spain.
}

Thruster (RIT), Helicon ${ }^{5,7-10}$ or electron cyclotron resonance thrusters, ${ }^{2}$ as well as very low power micropropulsion technologies for the precise positioning of nanosatellites such as the electrospray or colloid thrusters. ${ }^{6}$

The above conditions are met by low electric power versions of gridded ion engines ${ }^{1,2}$ (GIE), Hall effect thrusters ${ }^{1,2}$ (HET), and multi-cusped field thrusters ${ }^{2,11-15}$ (MCFT) such as the highly efficient multistage plasma thruster ${ }^{14,15}$ (HEMPT). The characteristics of these propulsive systems have been widely discussed in textbooks ${ }^{1}$ and comprehensive reviews. ${ }^{2-6}$

In this paper, we report the characteristics of supersonic plasma jets produced by the DC powered plasma thruster in Fig. 1 in connection with its operation modes. This alternative low power hybrid ion engine (ALPHIE) concept of a gridded plasma accelerator ${ }^{16}$ is different from those previously mentioned as well as those in our previous works. ${ }^{17}$

As we shall see, the suprathermal speed of the plasma jet is voltage controlled, and this feature is crucial for space propulsion applications. The subsequent expansion of this plasma beam within the limited volume of our vacuum chamber produces plasmas with peculiar properties. High ion temperatures result in electron Debye lengths $\lambda_{D e} \ll \lambda_{D i}$ shorter than the ion Debye lengths. Two kinds of ionizing collisions could coexist because the cross-sections of ion and electron impact by fast electrons become of the same order due to the high energies of ions, similar to the cathode dark space of DC glow discharges. ${ }^{18}$ 


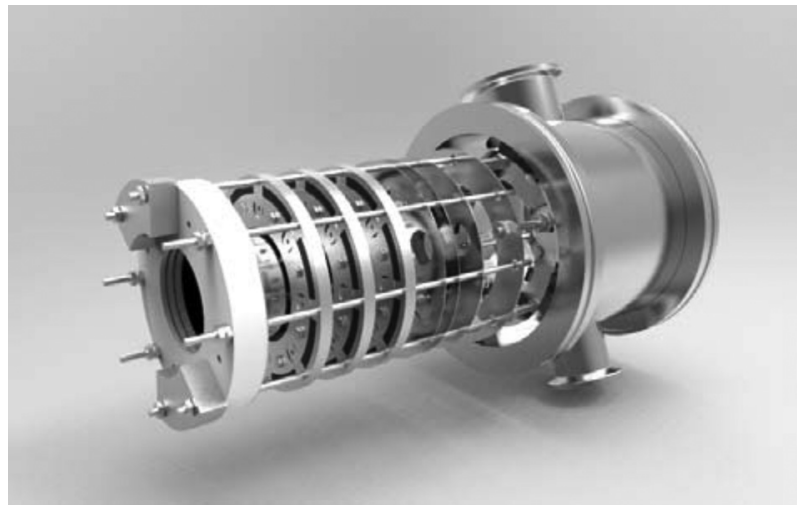

FIG. 1. Engineering drawing of the ALPHIE plasma thruster with its DN100 ISO-K vacuum flange. The front grids, the cathode wire and the magnetic insulation casing are not shown in the picture.

This paper is organized as follows: the ALPHIE conception is discussed in Sec. II, the details of the experimental setup for testing its performance and operation are in Secs. III and IV, whereas the working modes are discussed in Sec. V. The supersonic plasma streams are investigated in Sec. VI using the ion energy distribution functions (IEDF). We end at Sec. VII with some concluding remarks and the estimate of the performance characteristics of our plasma engine.

\section{THE ALPHIE PLASMA THRUSTER}

Figure 2 shows the cross-sectional scheme along the axial direction of symmetry of the small $(10 \mathrm{~cm}$ diameter and $14 \mathrm{~cm}$ length) plasma engine shown in Fig. 1. The stainless steel cylindrical plasma or ionization chamber has a diameter $D=4 \mathrm{~cm}$ and a length of $10 \mathrm{~cm}$, with one open end. To the front of its aperture are two parallel metallic (molybdenum) grids which will be denominated ion extraction and cover grids.

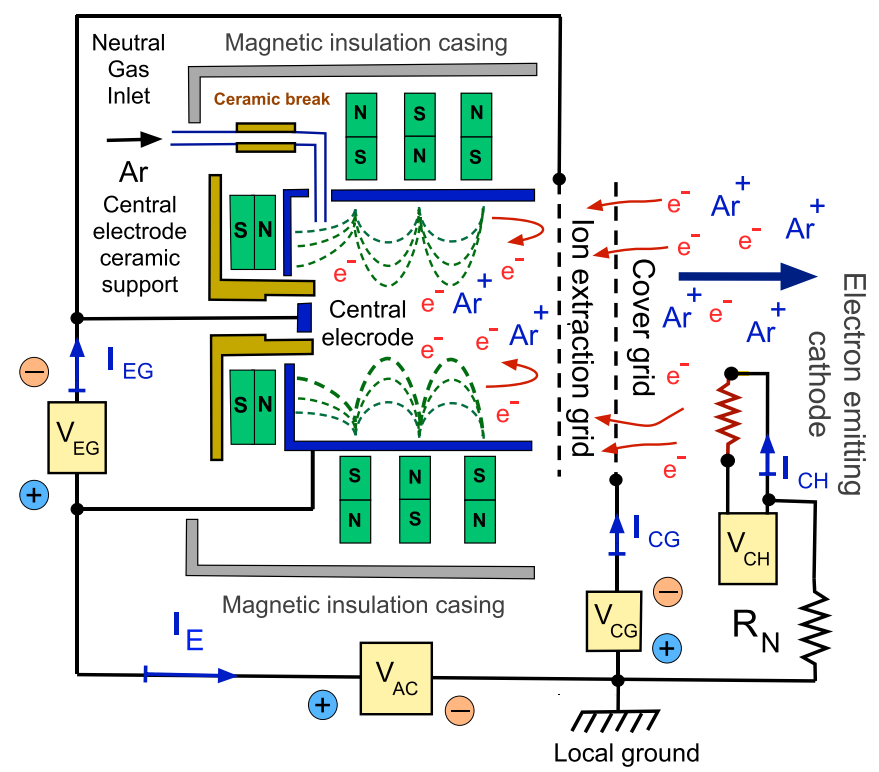

FIG. 2. Electrical connection scheme of the plasma thruster, where $R_{N}=1.5 \Omega$. The only electron-emitting cathode is employed for both plasma formation and ion beam neutralization. The dotted lines indicate the magnetic field pattern inside the ionization chamber, which is generated by the alternate polarity of permanent magnets placed outside.
The molybdenum grid meshes, with $57.8 \%$ open area and $0.97 \mathrm{~mm}$ wire diameter, have their apertures aligned to facilitate the flow of charged particles through them. The grids (at $2.0 \mathrm{~mm}$ separation) are electrically insulated from other elements by ceramic spacers.

The propellant neutral gas (argon or xenon) is introduced into the plasma chamber through a $3 \mathrm{~mm}$ diameter pipe connected to the ceramic break connector indicated in Fig. 2. The gas feed system is thus electrically insulated from the plasma thruster body.

The only electron source in the scheme of Fig. 2 is placed outside the plasma chamber. The electron-emitting cathode can have different forms, such as hollow cathodes, dispenser cathodes, or thermionic electron emitters. This electron source is used for both ionization of the neutral gas and neutralization of the outflowing ion beam.

In our case, the electron source is a thoriated tungsten wire of $0.25 \mathrm{~mm}$ diameter that is heated up to thermionic electron emission by a DC current. With this choice, the plasma engine is the only ion source in the experiments discussed in Secs. V and VI.

The central electrode and the ion extraction grid at the front (open end) of the ionization chamber are electrically connected. The central metallic disk of $5 \mathrm{~mm}$ diameter is electrically insulated from the ionization chamber walls by a ceramic support, which also ensures that it is symmetric with the axis of the system.

The DC voltage $V_{E G}$ (typically $100-200 \mathrm{~V}$ ) positively biases the conductive walls of the ionization chamber with respect to the central electrode. Thus, electrons already inside the chamber that would flow towards its open end are returned back by the ion extraction grid, whereas the massive ions are attracted towards its open spaces and to the small central electrode. The current $I_{E G}$ measures the flow of charges between the metallic chamber walls and the two connected electrodes. As we shall see, the current $I_{E G}$ is minimal because of the low capture of ions by the extraction grid and at the central electrode.

Figure 2 shows the positive DC voltage $V_{A C}$ (typically $400-650 \mathrm{~V}$ ) used to electrically bias the external cathode with respect to the conductive walls of the plasma chamber. This electric potential attracts a fraction of electrons emitted by the cathode inwards through the open spaces of the grids. The ions are accelerated to supersonic speeds in the opposite direction by the potential $V_{A C}$.

The electric potential of the cover grid is usually set to null $\left(V_{C G}=0\right)$, so that it is at a potential equal to that of the electron-emitting cathode and the thruster electrical ground. For large negative voltages $V_{C G}$, the cover grid repels the ionizing electron inflow, and can be used to interrupt the electric charge production. Additionally, the current $I_{C G}$ in Fig. 2 is also small because of the low capture of electrons and ions by the thin wire mesh.

The magnetic field inside the ionization chamber is produced by three concentric crowns made of eight permanent magnets each and an additional ring-shaped magnet placed at its closed end. The stainless steel plasma chamber is essentially transparent to the magnetic field produced by these high-temperature magnets placed outside. They were 
manufactured with a precise circular shape from a samarium cobalt alloy (SmCo 2:17), and have a surface magnetic induction of about $350 \mathrm{mT}$.

These magnets are fixed with bolts to the structure shown in Fig. 1 to keep them separated by $0.5 \mathrm{~mm}$ from the external surface of the plasma chamber. The poor thermal contact ensures the thermal stability of the magnets, and their deterioration is minimal since they are not exposed to the plasma.

As shown in Fig. 2, the magnets have alternate polarities along the axial and tangential directions, with the pole of each magnet opposite to that of the adjacent one. The magnetic field lines bend inside the plasma chamber, connecting the surfaces of the neighbor magnets placed outside along both axial and radial directions. This magnetic field configuration is intended to produce the turbulent motion of confined ionizing electrons inside the plasma chamber, contrary to the usual MCFT schemes.

The measured magnetic field strength along the inner surface of the chamber reaches a maximum value of $150-250 \mathrm{mT}$ at the cusps near the magnets. It decreases to 25-50 $\mathrm{mT}$ at the interspace between two successive magnets. The lowest values, 1-10 mT, were measured along the centerline of the ionization chamber.

The energy of the ionizing electrons from the outside cathode within the plasma chamber is driven by the voltage $V_{A C}$ and lies in the range $400-600 \mathrm{eV}$. Then, the typical gyroradii are $r_{e} \sim 0.2-0.4 \mathrm{~mm}$ for points close to the cusps, increasing to $r_{e} \sim 2.0-4.0 \mathrm{~mm}$ in the space between two consecutive magnets. Close to the centerline of the plasma chamber, the values increase to $r_{e} \sim 6.0-60 \mathrm{~cm}$. The diameter of the ionization chamber $D$ is thus essentially smaller than the minimum Larmor radius $r_{i} \simeq 5 \mathrm{~cm}$ for singly charged argon ions.

Thus, the electrons are magnetized inside the ionization chamber, whereas the motion of heavy ions remains predominantly driven by the electric fields. The ionization rate increases because the electron drift motion is confined by the banana-shaped magnetic field lines along the tangential and axial directions inside the plasma chamber. Damage and sputtering produced over the inner surface of the plasma chamber is limited to the magnetic field cusp regions. Finally, a magnetic-field-proof casing encloses the thruster body to protect the nearby equipment from intense fields of the plasma thruster magnets.

The plasma thruster operates as follows: Setting $V_{C G}=0$, the ionizing electrons emitted by the outside cathode enter into the plasma chamber and are further accelerated inside by the voltages $V_{A C}$ and $V_{E G}$ to high energies above the ionization threshold of the propellant neutral gas. The electrons are additionally confined within the intricate pattern of magnetic field lines. This combination of electron trapping and acceleration increments the collisions with neutral atoms and the ionization rate of the neutral gas. Plasma is produced without an electron-emitting cathode disposed inside the ionization chamber, contrary to GIE thruster schemes.

Next, positive ions are attracted towards the open end of the plasma chamber by the negative voltage $V_{E G}$ applied to the extraction grid. On the contrary, the electrons are collected at the plasma chamber walls. Finally, this ion outflow is further accelerated by the potential $V_{A C}$ through the open spaces of the cover grid. This ion outflow is not space charge limited because of the ionizing electron inflow through the grids as shown in Fig. 2.

As we shall see in Secs. V and VI, the thruster current $I_{E} \gg I_{C G} \simeq I_{E G} \simeq 0$ due to the low ion and electron captured at the grids compared to the electron current collected at the plasma chamber walls.

Finally, additional electrons from the external cathode neutralize the ion beam, and the resulting plasma stream moves in the direction indicated by the thick arrow in Fig. 2.

\section{EXPERIMENTAL SETUP}

The performance of the ALPHIE plasma engine discussed in Sec. II was tested in a cylindrical stainless steel vacuum tank of $80 \mathrm{~cm}$ length and $40 \mathrm{~cm}$ diameter. The base pressure of argon was $5 \times 10^{-6}$ mbar and the working pressures $p_{a}$ were in the range $4-8 \times 10^{-5}$ mbar for gas flow rates $\mathrm{Q}=0.5-1.5$ standard cubic centimeters by minute $(\mathrm{sccm})$ injected into the plasma chamber. These are equivalent to argon mass flow rates $\dot{m}=0.89-2.7 \mathrm{mg} / \mathrm{min}$.

These flow rates were held constant by using a Bronkhorst F201C valve and an E-7100 flow controller. The corrections for the neutral gas backflow from the vacuum tank through the open spaces of the grids were also considered. ${ }^{1}$ Table I shows the characteristics of DC power supplies employed during the experiments. Additionally, the local ground of the plasma thruster and its power supplies in Fig. 2 were electrically insulated from the grounded walls of the vacuum tank. Thus, the stream of charged particles is the only connection between the plasma engine and the diagnostic equipment that were electrically independent.

\section{PLASMA DIAGNOSTICS}

The properties of plasma streams were determined using a collecting Langmuir probe (LP) and a four-grid retardingfield energy analyzer (RFEA). Both diagnostics were positioned, in turn, along the axis of symmetry at a fixed point, $20 \mathrm{~cm}$ distance from the exit section of the plasma thruster.

The spherical stainless steel LP has a diameter of $4.44 \mathrm{~mm}$, and was biased using a sweep circuit with respect to the grounded walls of the vacuum tank. ${ }^{19-21}$ This LP probe was also employed to monitor the eventual presence of plasma waves and oscillations during the measurements with the RFEA.

Electron densities of $n_{e} \simeq 0.1-8.0 \times 10^{8} \mathrm{~cm}^{-3}$ with electron temperatures of $T_{e}=1-2 \mathrm{eV}$ were measured at distant points from the plasma thruster where the LP characteristic

TABLE I. The characteristics of the DC power supplies of the electrical scheme of Fig. 2 employed in the experiments.

\begin{tabular}{lcc}
\hline \hline Cathode heating & $150 \mathrm{~V}, 10 \mathrm{~A}$ & Agilent N5770A \\
Ion extraction $V_{E G}$ & $150 \mathrm{~V}, 10 \mathrm{~A}$ & Agilent N5770A \\
Acceleration $V_{A C}$ & $1.5 \mathrm{kV}, 0.75 \mathrm{~A}$ & Fug MCA 750-1500 \\
Cover grid $V_{C G}$ & $300 \mathrm{~V}, 5 \mathrm{~A}$ & Agilent N5771A \\
\hline \hline
\end{tabular}


curves approximately correspond to a Maxwellian plasma. The corresponding electron Debye lengths $\lambda_{D e}$ are between $0.2 \mathrm{~mm}$ and $3.3 \mathrm{~mm}$.

The one-dimensional ion energy distribution functions $f_{i}(E)$, where $E=m_{i} u_{z}^{2} / 2$ and $u_{z}>0$ is the ion speed parallel to the RFEA axis of symmetry, are obtained from the voltage-current characteristic curves. ${ }^{22,23}$ This diagnostic was operated in the steady state and has four equally spaced grids separated by $d=6.26 \mathrm{~mm}$ and placed transversal to a cylindrical open channel of $25.05 \mathrm{~mm}$ length and $4.0 \mathrm{~mm}$ diameter for ion motion. The molybdenum grid meshes have an open area of $87 \%$, a wire diameter of $0.0509 \mathrm{~mm}$, and an average opening width of $0.068 \mathrm{~mm}$. The grids were carefully aligned. However, we lack the quantitative measurement of the particle transmission rate through the ion channel. The copper ion collector plate is also separated by $6.26 \mathrm{~mm}$ from the electron suppressor grid and closes the back end of the RFEA ion channel.

The grids are considered as equipotential surfaces because the size of their open spaces are below the characteristic electron Debye lengths. Additionally, space charge effects can be neglected because the separation between consecutive grids is less than two electron Debye lengths $d \lesssim 2 \lambda_{D e} .^{22,23}$

Under these conditions, the motion of ions inside the open channel is collisionless and only affected by the electric potentials imposed upon the grids. ${ }^{23}$ The first entrance mesh in contact with the plasma was electrically floating, whereas the plasma electron repeller and secondary electron suppressor grids were typically biased to $-30 \mathrm{~V}$ with respect to ground. The ion current $I_{c}=S \times J_{c}\left(V_{i d s}\right)$ collected by the RFEA back plate (surface $S=12.6 \mathrm{~mm}^{2}$ ) was measured as a function of the ion discriminator grid voltage $V_{i d}{ }^{22,23}$

These RFEA current-voltage (IV) characteristic curves were digitized using a Keithley 6487 picoammeter that sweeps $V_{i d}$ between $\pm 500 \mathrm{~V}$ in 1000 fixed steps of $1 \mathrm{~V}$ with respect to the (grounded) walls of the vacuum chamber. A typical IV curve is shown in Fig. 3, where the maximum ion current $I_{c}\left(V_{i d}\right)$ corresponds to low ion-repelling potentials $V_{i d}$ that filter high energy ions as the ion discriminator potential increases. ${ }^{22,23}$ No relevant plasma waves or oscillations were observed from the Langmuir probe measurements.

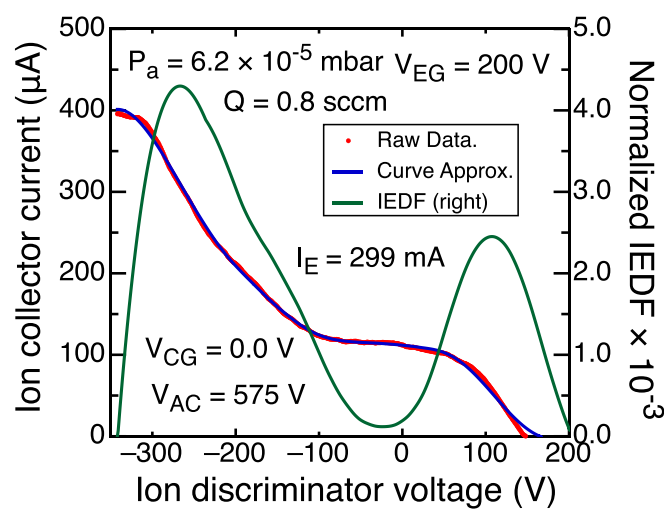

FIG. 3. The RFEA raw data (dots) with the numerical (Savitzky-Golay) approximation of the IV curve (solid curve). The calculated IEDF refers to the right axis.
The current density of singly charged ions at the RFEA end plate is $J_{c}=e u_{z} d n_{i}$, where $d n_{i}=f_{i}\left(u_{z}\right) d u_{z}$ is the number of ions with axial speeds between $u_{z}$ and $u_{z}+d u_{z}$ and $e>0$ is the electron charge. ${ }^{22,23}$ The integral

$$
J_{c}\left(e V_{i d}\right)=e \int_{u_{m z}}^{\infty} u_{z} f_{i}\left(u_{z}\right) d u_{z}=\frac{e}{m_{i}} \int_{e V_{i d}}^{\infty} f_{i}(E) d E
$$

gives the collected ion current density of ions with energies $E=m_{i} u_{z}^{2} / 2 \geq e V_{i d} .{ }^{22}$ As is predicted in Eq. (1), the ion current in Fig. 3 monotonically decreases from a maximum value $J_{c}\left(e V_{m}\right) \geq J_{c}\left(e V_{i d}\right)>0$ when all ions of energy distribution $f_{i}(E)$ reach the RFEA end plate.

In Eq. (1), the potential $V_{m}$ corresponds to the lowest possible speed $u_{m z} \simeq 0$ detected by the RFEA and is different from zero because $V_{i d}$ is referenced to ground instead of the plasma potential. Figure 3 shows $f_{i}\left(e V_{i d}\right)$ against the actual ion discriminator grid voltage whereas the ion energy $E=e$ $\left(V_{i d}-V_{m}\right)>0$ is used in Figs. 6-8. The RFEA ion energy resolution is about $\Delta E \pm 4 \mathrm{eV}$, equivalent to axial speeds of $\pm 4.4 \mathrm{Km} / \mathrm{s}$ for singly charged argon ions with energies below $650 \mathrm{eV}$. This minimum axial velocity is similar to typical ion sound speeds of the low energy ion population.

Differentiating Eq. (1), we get

$$
\frac{d J_{c}}{d V_{i d}}=-\frac{e^{2}}{m_{i}} f_{i}\left(e V_{i d}\right)
$$

and we make use of this equation to obtain the distribution function $f_{i}(E)$ from the IV curves of the RFEA. The typical IV curves have 800-900 equally spaced data points which were numerically approximated using the Savitzky-Golay numerical scheme to evaluate their derivatives. ${ }^{24,25}$ Figure 3 shows a typical IV curve along with its numerical approximation and its derivative, proportional to the IEDF which refers to the right axis.

The two slopes in the IV curve give rise to two peaks in the IEDF of Fig. 3, which can be seen as a superposition of two ion populations. The high energy peak corresponds to the suprathermal (or supersonic) ion population that drifts with respect to the low energy ion group. Similar IV characteristic curves and IEDFs have been obtained in the characterization of supersonic ion beams with gridded RFEAs in contexts as different as in plasma-assisted material modification, ${ }^{26}$ double layers, ${ }^{27}$ plasma jets from GIEs, ${ }^{28,29}$ double layer plasma thrusters, ${ }^{7,8,30}$ or experiments with helicon plasma sources. $^{31-33}$

\section{OPERATION OF THE PLASMA THRUSTER}

The typical values of magnitudes of parameters indicated in the scheme of Fig. 2 for the operation of the ALPHIE plasma thruster are shown in Table II. In our experiments, we limited $I_{E}$ to $100-500 \mathrm{~mA}$ to maximize the ion production outflow within the technical limits of our equipment. Specifically, the mass flow rates $Q \leq 1.5 \mathrm{sccm}$ and $P_{C H}$ $=V_{C H} \times I_{C H}<76 \mathrm{~W}$, which bounded the ion production. The maximum thruster electric power was $P_{T}=V_{A C} \times I_{E}$ $=325 \mathrm{~W}$, whereas $P_{E G}=I_{E G} \times P_{E G}$ and $P_{E C}=I_{E C} \times P_{E C}$ 
TABLE II. The operational parameters of the scheme of Fig. 2 in the experiments discussed in Secs. V and VI.

\begin{tabular}{lcc}
\hline \hline Pressure & $4.0-10.0 \times 10^{-6} \mathrm{mbar}$ & \\
Argon flow & $Q=0.5-1.5 \mathrm{sccm}$ & \\
Acceleration & $V_{A C}=400-650 \mathrm{~V}$ & \\
Thruster current & $I_{E}=100-500 \mathrm{~mA}$ & $I_{C G} \leq 2 \mathrm{~mA}$ \\
Cover grid & $V_{C G}=0-350 \mathrm{~V}$ & $I_{E G} \leq 2 \mathrm{~mA}$ \\
Ion extraction grid & $V_{E G}=100-300 \mathrm{~V}$ & $I_{C H}=5.5-6.3 \mathrm{~A}$ \\
Cathode heating & $V_{C H}=10-12 \mathrm{~V}$ & \\
Max. power & $<450 \mathrm{~W}$ & \\
\hline
\end{tabular}

were much lower. Thus the overall DC electric power $P_{T}+P_{C H}+P_{E G}+P_{C G}$ was always below 450 Watts.

The operation of the ALPHIE plasma engine follows three steps: First, the voltages $V_{A C}$ and $V_{E G}$, and the propellant gas flow rate $Q$ are fixed to values within the ranges indicated in Table II. Next, the potential $V_{C G}$ is set to null, to maximize the ionizing electron inflow towards the ionization chamber. Finally, the DC heating power of the external cathode is increased to thermionic electron emission. ${ }^{34}$

This increment in the $P_{N H}$ multiplies the high-energy electrons attracted and trapped inside the ionization chamber. The thruster current $I_{E}$ increases with the rising ionization rate, and for $I_{E} \approx 100 \mathrm{~mA}$, the plasma outflow can be detected by the Langmuir probe within the vacuum chamber. Under these conditions, the currents $I_{C G}$ and $I_{E G}$ are always below $2 \mathrm{~mA}$, two orders of magnitude lower than $I_{E}$ because of the low loss rate of charged particles due to collection by the grids.

The thruster current $I_{E}$ is mainly composed of electrons collected at the plasma chamber walls and roughly characterizes the charge production inside. It depends on three parameters, namely the acceleration potential $V_{A C}$, the propellant mass flow rate $Q$, and the inflow of ionizing electrons from the cathode. The latter is governed in our case by the temperature of the hot cathode wire of Fig. 2, which is determined by the heating power $P_{C H}{ }^{34}$ These electrons control both the ion production within the plasma chamber and the space charge neutralization.

Figures 4 and 5 show the operation curves of this plasma engine for a constant thermionic electron emission rate (or equivalently, a fixed power $\left.P_{C H}\right)$. The thruster current $I_{E}$ that

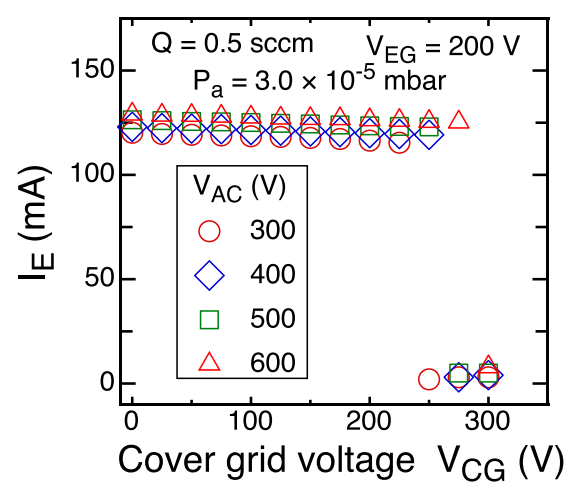

FIG. 4. The thruster current $I_{E}$ as a function of the cover grid voltage $V_{C G}$ for different acceleration voltages $V_{A C}$ holding the gas flow rate $Q$ constant.

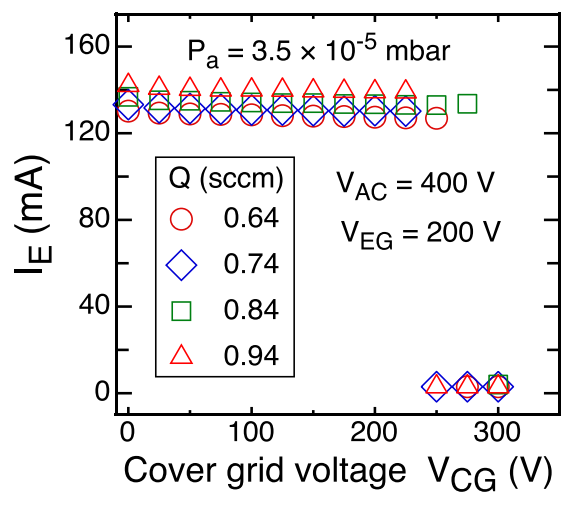

FIG. 5. The thruster current $I_{E}$ as a function of the cover grid voltage $V_{C G}$ for different gas flow rates $Q$ holding the acceleration voltage constant.

measures the production of charges inside the plasma chamber is represented against the cover grid voltage $V_{C G}$. Higher ion production rates can be achieved using either increased acceleration voltages $V_{A C}$ for ionizing electrons or incremented mass flow rates $Q$ of the injected propellant.

In Fig. 4, the current $I_{E}$ grows from $120 \mathrm{~mA}$ to $130 \mathrm{~mA}$ when the acceleration voltage $V_{A C}$ is increased from $300 \mathrm{~V}$ to $600 \mathrm{~V}$, holding the mass flow rate $Q=0.5 \mathrm{sccm}$ constant. A similar increase in $I_{E}$ from 130 to $140 \mathrm{~mA}$ is observed in Fig. 5, where $V_{A C}=400 \mathrm{~V}$ was fixed, whereas the mass flow rate $Q$ grows from 0.64 to $0.94 \mathrm{sccm}$.

Furthermore, in both Figs. 4 and 5, the current $I_{E}$ abruptly decreases by more than an order of magnitude to 3-8 $\mathrm{mA}$ for $V_{C G}$ above a certain threshold value. This reduction occurs because a large fraction of the thermionic electrons are repelled by the cover grid. The potential $V_{C G}$ hinders the inflow of ionizing electrons and the ion production, and thus $I_{E}$ abruptly falls. In our experiments, the potential $V_{C G}$ was set to null, biasing the cover grid to the local ground of Fig. 2, hence maximizing the ion outflow.

\section{SUPERSONIC ION BEAMS WITH CONTROLLED SPEED}

The IEDFs of the ALPHIE plasma streams are waterfall displayed in three dimensions in Figs. 6-8 under different operational conditions. These IEDF curves were calculated as in Fig. 3 from measurements with the RFEA at a fixed point along the symmetry axis.

Using thermionic electron emission, the plasma thruster is the only source of ions in our experiments. This avoids the eventual distortion of the IEDFs caused by the presence of ions from other plasma sources, as can be the case when additional plasma from a hollow cathode is employed for ion beam neutralization.

The two-peaked normalized IEDFs for different acceleration potentials $V_{A C}$, at a constant thruster current $I_{E}$, are shown in Figs. 6 and 7. These ion energy spectra show the axial speed of the supersonic plasma stream to be governed by the acceleration potential $V_{A C}$ in the scheme of Fig. 2. In order to stress this heavy particle acceleration effect, these IEDFs are represented for decreasing the acceleration voltages $V_{A C}$. 


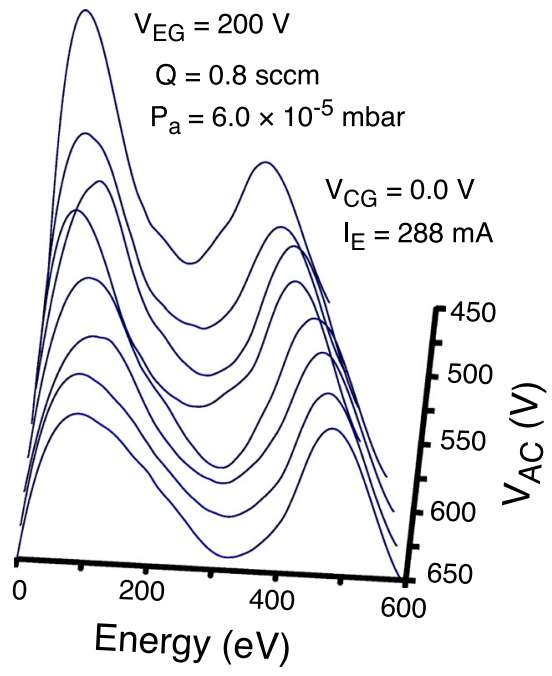

FIG. 6. Waterfall representation of the normalized IEDF against the acceleration voltages $V_{A C}=450,500,525,550,575,600,625$, and $650 \mathrm{~V}$ for a constant thruster current $I_{E}=288 \mathrm{~mA}$.

The low-energy peaks of these distributions are always centered around $E_{S i} \simeq 100 \mathrm{eV}$, and these heavy particles have lost a large fraction of their initial energies in the plasma expansion process. The fast ion groups have peak energies in the range $E_{F i} \simeq 350-550 \mathrm{eV}$, which linearly depend on the acceleration voltage in Figs. 6 and 7 . Therefore, the energy of this suprathermal ion population is controlled by the voltage $V_{A C}$ of the scheme in Fig. 2 .

This feature is clearly seen in Fig. 9, where the energies corresponding to the IEDF maxima in Figs. 6 and 7 are represented against the acceleration voltage. The maximum speeds of the low energy ion population corresponding to $E_{S i}$ $\simeq 100 \mathrm{eV}$ are insensitive to $V_{A C}$. However, the energies $E_{F i}$ corresponding to the maxima of the fast ion groups linearly increase with this acceleration potential. At the distant point where the RFEA is located, the high speed ion population still retains its original energy imparted by the acceleration potential $V_{A C}$.

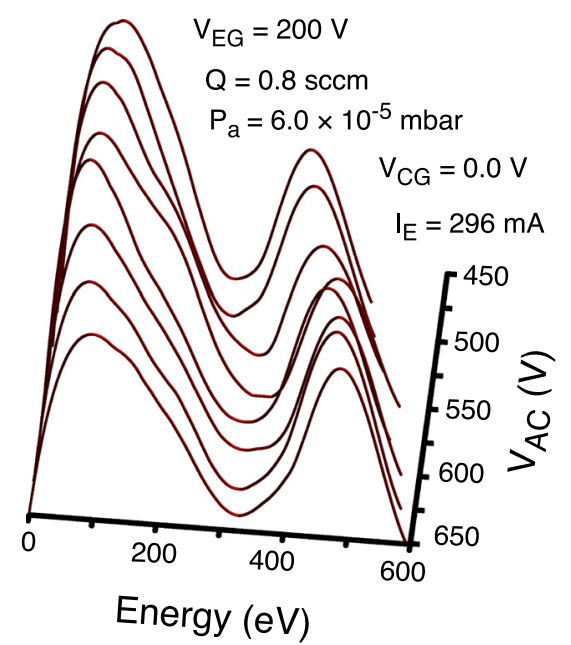

FIG. 7. Waterfall representation of the normalized IEDF against acceleration voltages $V_{A C}=450,475,500,550,575,600,625$, and $650 \mathrm{~V}$ for the constant thruster current $I_{E}=296 \mathrm{~mA}$.

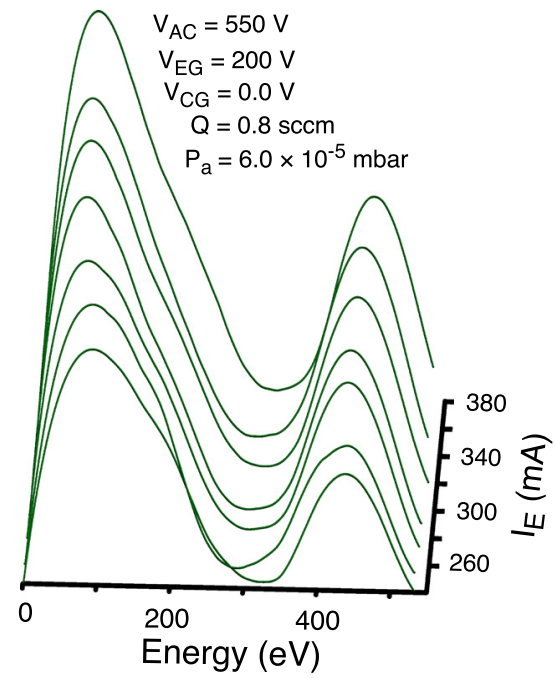

FIG. 8. Waterfall representation of the IEDFs (in arbitrary units) as a function of the currents $I_{E}=241,255,274,291,321,340$, and $380 \mathrm{~mA}$ holding the plasma acceleration voltage constant at $V_{A C}=550 \mathrm{~V}$.

In Fig. 9, the peak drift velocity $u_{D F}=\sqrt{2 \Delta E / m_{i}}$ of the fast ion group is estimated using the difference $\Delta E=E_{F i}-E_{S i}$ between the energies of the maxima (solid symbols) of the fast $\left(E_{F i}\right)$ and slow $\left(E_{S i}\right)$ ion groups. These velocities (open symbols in Fig. 9) are in the range $u_{D F}=36.6-43.5 \mathrm{Km} / \mathrm{s}$ and linearly depend on $V_{A C}$.

The effective temperatures of both ion groups in each case could be estimated by approximating the IEDFs in Figs. 6 and 7 by a sum of one to three analytical Gaussian functions. The calculation of the full width at half maximum (FWHM) of the peaks gives $T_{S i} \simeq 80-90 \mathrm{eV}$ for the low energy ion population and $T_{F i} \simeq 50 \mathrm{eV}$ for the fast ion group.

Then, the typical ion thermal speeds for the low energy population are $u_{S i} \simeq 20-21 \mathrm{Km} / \mathrm{s}$ and $u_{F i} \simeq 16 \mathrm{Km} / \mathrm{s}$ for the fast energy ion group. The characteristic electron thermal speeds are $u_{T e} \sim 593-836 \mathrm{Km} / \mathrm{s}$, much higher than $u_{S i}, u_{F i}$ and the drift speed $u_{D F}$ of fast ions.

Furthermore, the relative weight of high-energy ions in the IEDF also increases with $V_{A C}$. The slow ion group maxima in Figs. 6 and 7 decrease with $V_{A C}$, whereas the high-energy peaks grow. In Fig. 6, about $65.2 \%$ of ions have energies

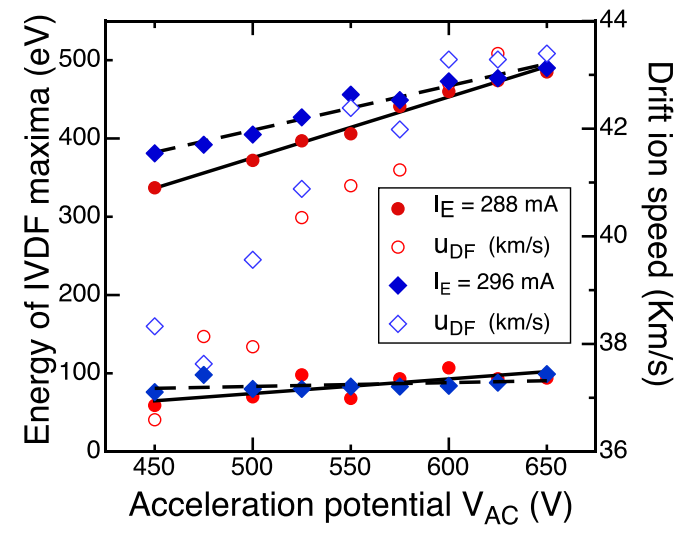

FIG. 9. The energies $E_{S i}$ and $E_{F i}$ of IEDF maxima (solid symbols) of Figs. 6 and 7 against the acceleration voltage $V_{A C}$ with their best linear fits. The corresponding speeds $u_{D F}$ of the fast ion group (open symbols) refer to the right axis. 
below that corresponding to the IEDF minimum, whereas $34.8 \%$ have higher energies for $V_{A C}=450 \mathrm{~V}$. These figures change to $59.5 \%$ and $40.5 \%$ when $V_{A C}=650 \mathrm{~V}$. The number of fast ions reaching the distant RFEA thus grows with $V_{A C}$.

Unfortunately, we are not in position to measure the ion densities of the plasma streams with our RFEA. However, the non-normalized IEDFs in Fig. 8 provide a qualitative assessment of the relation between the increase in the plasma beam current and the thruster current $I_{E}$ with the ionizing electron inflow. The latter is controlled by the DC heating power $P_{C H}$, which regulates the temperature of the cathode wire $^{34}$ in the scheme of Fig. 2.

The IEDFs in Fig. 8 are shown for different thruster currents $I_{E}$ while holding the acceleration potential at $V_{A C}$ $=550 \mathrm{~V}$. Figure 10 shows the linear dependencies on $P_{C H}$, of the thruster current $I_{E}$ (solid squares) and the energies of IEDF maxima (open symbols) in Fig. 8.

The heights of peaks in Fig. 8 increase with $P_{C H}$ and Fig. 10 shows the growth rate to be similar for both slow and fast ion populations. However, the energies corresponding to the IEDF maxima $\left(E_{S i} \simeq 100 \mathrm{eV}\right.$ and $E_{F i} \simeq 450 \mathrm{eV}$ for slow and fast ion groups, respectively) remain unaffected when the acceleration voltage $V_{A C}$ is kept constant.

When raising $P_{C H}$ at a fixed $V_{A C}$, the plasma engine produces a denser ion beam because of the incremented inflow of ionizing electrons into the plasma chamber. Consequently, the plasma beam density grows within the vacuum chamber as well as the heights of the peaks of IEDFs in Fig. 8.

The enhanced thermionic electron current also contributes to the rapid growth of the thruster current $I_{E}$ with $P_{C H}$ in Fig. 10 (right axis). This enhancement reflects the increment of electrons inside the plasma chamber, the ion production rate and the counter flow of charges with opposite signs through the grids; such a flow is not space-charge limited.

\section{CONCLUSIONS}

The experimental results discussed in Sec. VI show that the ALPHIE plasma engine produces a mesothermal flow where the supersonic plasma jet moves at intermediate drift speeds $u_{D F}$ between the electron and ion thermal speeds $u_{T e}$ and $u_{S i}$, respectively.

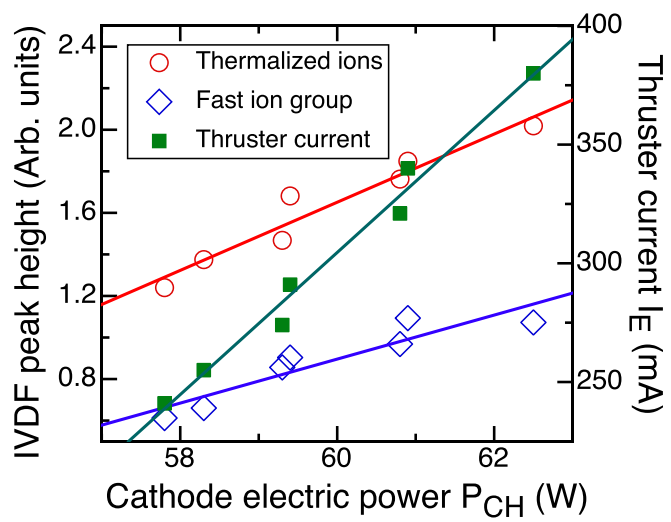

FIG. 10. The IEDF maxima in Fig. 8 (open symbols) and the thruster current $I_{E}$ (solid squares) with their best linear fits represented against the heating power $P_{C H}$ of the cathode wire of Fig. 2.
The IEDFs in Figs. 3 and 6-8 only account for the ion velocity component $u_{z}$ along the axis of symmetry of the RFEA. Figure 9 shows the acceleration potential $V_{A C}$ to drive the fast ion velocity along this axial direction. The low energy ion group is insensitive to $V_{A C}$ suggesting that their velocities have an isotropic thermal distribution without a preferred direction, contrary to the high energy group.

We have found the fast ion groups in Figs. 6-8 drift along the direction of the plasma jet with respect to slow ions at speeds $u_{D F} \simeq 36.6-43.5 \mathrm{Km} / \mathrm{s}$ that mainly depend on the acceleration potential $V_{A C}$ of Fig. 2. These fast ion speeds (open symbols in Fig. 10) are higher than the characteristic ion thermal speeds $u_{F i}$ and $u_{S i}$ and similar to plasma flows produced by other plasma thrusters. ${ }^{7,8,29-31,33}$

Furthermore, two collisional ionization processes may coexist downstream of the thruster grids. The voltage $V_{A C}$ of Fig. 2 imparts energies in the range $400-650 \mathrm{eV}$ to the thermionic electrons that face the slow neutral gas outflow from the ionization chamber through the grid holes. The electron impact ionization rate is orders of magnitude higher for these drifting electrons compared to ion production by the cold $\left(T_{e}=1-2 \mathrm{eV}\right)$ electron group away from the plasma engine. $^{35}$

Additionally, the values of cross-sections ${ }^{36}$ for argon ionization by ion impact

$$
A r^{+}(\text {fast })+A r(\text { slow }) \rightarrow A r^{+}(\text {fast })+A r^{+}(\text {slow })+e^{-}
$$

for both ion groups $\left(0.5-1 \times 10^{-16} \mathrm{~cm}^{2}\right)$ become comparable to those of ionization by electron impact $\left(1-3 \times 10^{-16} \mathrm{~cm}^{2}\right)$. As for electrons, the high energy ion group with energies in the range $300-600 \mathrm{eV}$ in Figs. 6-8 would increase the ion impact ionization rate as in the dark space of glow discharges. ${ }^{18}$

The low energy ion peaks in Figs. 6-8 originated from collisional energy losses of fast ions in the plasma jet expansion. First, charge exchange collisions would have a dominant role in the production of slow ions from fast heavy charged particles. An additional source of low energy ions is ion impact collisions. Finally, the fast ions of Figs. 6-8 recombine at the walls of the vacuum chamber, but a significant fraction can be slowed by the metallic surfaces and later backscattered with lower energies. Similar two-peaked ion energy spectra have been observed in the plasma plume expansion process. $8,30-33$

These collisional energy losses of fast ions could be responsible for the high temperatures $T_{S i} \sim 80-90 \mathrm{eV}$ of the slow ion group, well over the typical electron temperatures $T_{e} \sim 1-2 \mathrm{eV}$. In consequence, the electron Debye lengths $\lambda_{D e}$ $\simeq 0.2-3.3 \mathrm{~mm}$ are smaller than the ion Debye lengths $\lambda_{D i}$ $\simeq 20-22 \mathrm{~mm}$ in the plasma far from the plasma engine.

Therefore, Figs. 6-10 show that the ALPHIE conception has the essential performance required of a plasma thruster, namely the generation of a supersonic ion flow with a speed controlled by the acceleration potential. Figs. 8 and 10 show how the electron emission current from the external cathode increases the ion production of both slow and fast ion groups. The plasma stream density and the thruster current $I_{E}$ grow with the ionizing electron inflow. 
The gridded plasma thruster discussed in Sec. II presents important differences from other GIE or Kaufman ion engine schemes. ${ }^{1,2}$ No additional cathode is required inside the plasma chamber for the ionization of the propellant gas, and the cover grid potential $V_{C G}$ is not responsible for ion acceleration, as shown in Figs. 4-8. The ionizing electrons and accelerated ions counter-flow through the grids, and thus this electric current is not space-charge limited. On the contrary, only ions are transported by the grids in GIE designs where the electron backstreaming from the external neutralizer cathode is considered a failure mechanism. ${ }^{1,2}$

The only electron-emitting cathode is disposed on the outside, and is employed for both plasma production and ion beam neutralization, as in HET and MCFT conceptions. This couples the plasma production and space charge neutralization processes. ${ }^{2,31,33}$ However, our plasma chamber is metallic as in GIEs, ${ }^{1,2}$ whereas those of MCTs and HETs are made of ceramic materials. ${ }^{1,2,14,15}$

Using only one cathode, the simple ALPHIE hybrid design has less demanding requisites for the electronic equipment compared to usual GIEs, and it would be well suited for small and medium-sized satellites with limited electric power and reduced propellant storage capacities.

Future works will address the physical mechanisms involved in the ion acceleration processes and study the spatial profiles of the plasma plume. Higher propellant flow rates $Q$, improvement in the ion optics, and optimized cathodes are required to boost the ion production rate as well as the attainable thrust levels. These would lead to further reduction in the electric power and larger thrust-to-power ratios. $^{1-4}$

\section{ACKNOWLEDGMENTS}

The authors are indebted to Mr. P. Argente for his careful artwork and fruitful discussions, and to $\mathrm{Mr}$. J. González for his useful suggestions. The technical assistance from Mr. F. Sánchez is also appreciated. Mr. J. Damba and Dr. S. P. Tierno are thankful to FPI and FPU, respectively, for Fellowship Programs. This work was funded by the Ministerio de Economía, Ciencia e Innnovación of Spain under Grant No. ESP2013-41078-R. The authors also acknowledge the financial support from Aernnova Aerospace SAU.

${ }^{1}$ D. M. Goebel and I. Katz, Fundamentals of Electric Propulsion. Ion and Hall Thrusters (John Wiley \& Sons, Hoboken, New Jersey, USA, 2008), Chap. 1, 6, and 7.

${ }^{2}$ S. Mazouffre, Plasma Sources Sci. Technol. 25, 033002 (2016).

${ }^{3}$ C. Charles, J. Phys. D: Appl. Phys. 42, 163001 (2009).

${ }^{4}$ E. Ahedo, Plasma Phys. Controlled Fusion 53, 124037 (2011).

${ }^{5}$ F. F. Chen, Plasma Sources Sci. Technol. 24, 014001 (2015).
${ }^{6}$ M. Keidar, T. Zhuang, A. Shashurin, G. Teel, D. Chiu, J. Lukas, S. Haque, and L. Brieda, Plasma Phys. Controlled Fusion 57, 014005 (2015).

${ }^{7}$ C. Charles and R. W. Boswell, Phys. Plasmas 11, 1706 (2004).

${ }^{8}$ C. S. Corr, J. Zanger, B. W. Boswell, and C. Charles, Appl. Phys. Lett. 91, 241501 (2007).

${ }^{9}$ S. Pottinger, C. Lappas, C. Charles, and R. W. Boswell, J. Phys. D: Appl. Phys. 44, 235201 (2011).

${ }^{10}$ S. N. Bathgate, M. M. M. Bilek, and D. R. McKenzie, Plasma Sci. Technol. 19, 083001 (2017).

${ }^{11}$ H. Liu, P. B. Chen, Q. Q. Sun, P. Hu, Y. C. Meng, W. Mao, and D. R. Yu, Acta Astronaut. 126, 35 (2016).

${ }^{12}$ P. Hu, H. Liu, Y. Gao, and D. Yu, Phys. Plasmas 23, 103517 (2016).

${ }^{13}$ N. A. MacDonald, V. C. Young, M. A. Cappelli, and W. A. Hargus, Jr., J. Appl. Phys. 111, 093303 (2012).

${ }^{14}$ G. Kornfeld, N. Koch, and H.-P. Harmann, in Proceedings of the 30th International Electric Propulsion Conference, Florence, Italy (2007), IEPC Paper No. 2007-108.

${ }^{15}$ N. Koch, M. Schirra, S. Weiss, A. Lazurenko, B. van Reije, J. Haderspeck, A. Genovese, P. Holtmann, R. Schneider, K. Matyash, and O. Kalentyev, in Proceedings of the 32th International Electric Propulsion Conference, Weisbaden, Germany (2011), IEPC Paper No. 2011-236.

${ }^{16}$ L. Conde, J. L. Domenech-Garret, J. M. Donoso, E. Del Río, and M. A. Castillo, "Plasma accelerator with modulated thrust," Patent application PCT/EP2015/074879 (2015).

${ }^{17}$ E. Criado, E. Roibás, S. P. Tierno, P. Rodríguez De Francisco, J. L. Domenech-Garret, J. M. Donoso, and L. Conde, Phys. Plasmas 19, 023505 (2012).

${ }^{18}$ A. Bogaerts and R. Gijbels, J. Appl. Phys. 78, 6427 (1995).

${ }^{19}$ O. Troll, L. Conde, E. Criado, J. M. Donoso, and G. Herdrich, Contrib. Plasma Phys. 50, 819 (2010).

${ }^{20}$ S. P. Tierno, J. L. Domenech-Garret, J. M. Donoso, D. Jennewein, G. Herdrich, S. Fasoulas, and L. Conde, IEEE Trans. Plasma Sci. 41, 695 (2013).

${ }^{21}$ N. Hershkowitz, in Plasma Diagnostics. Discharge Parameters and Chemistry, edited by O. Auciello and D. L. Flamm (Academic Press, 1989), Vol. 1, Chap. 3, pp. 113-182.

${ }^{22}$ C. Böhm and J. Perrin, Rev. Sci. Instrum. 64, 31 (1993).

${ }^{23}$ I. H. Hutchinson, Principles of Plasma Diagnostics (Cambridge University Press, New York, USA, 1990), p. 76.

${ }^{24}$ F. Magnus and J. T. Gudmundsson, Rev. Sci. Instrum. 79, 073503 (2008).

${ }^{25}$ A. Savitzky and M. J. E. Golay, Anal. Chem. 36, 1627 (1964).

${ }^{26} \mathrm{~V}$. V. Zhurin, Ion and Plasma Sources for Science and Technology (Wiley-VCH, Weinheim, Germany, 2011), Chap. 5, p. 73.

${ }^{27}$ S. Rupnik, J. Kovačič, M. Čerček, T. Gyergyek, V. Pohoata, G. Popa, C. Ionita, and R. Schrittwieser, in Proceedings of the 39th EPS Conference \& 16th International Congress on Plasma Physics (Stockholm, Sweden, 2012), EPS paper No. P5-157.

${ }^{28}$ Z. Zhang, H. Tang, Z. Zhang, J. Wang, and S. Cao, Rev. Sci. Instrum. 87, 123510 (2016).

${ }^{29}$ Z. Zhang, H. Tang, J. Ren, Z. Zhang, and J. Wang, Rev. Sci. Instrum. 87, 113502 (2016).

${ }^{30}$ C. Charles, B. W. Boswell, and M. A. Lieberman, Appl. Phys. Lett. 89, 261503 (2006).

${ }^{31}$ L. T. Williams and M. L. R. Walker, IEEE Trans. Plasma Sci. 43, 1694 (2015).

${ }^{32}$ N. Gulbrandsen, ̊. Fredriksen, J. Carr, and E. Scime, Phys. Plasmas 22, 033505 (2015)

${ }^{33}$ M. Wiebold, S. Yung-Ta, and J. E. Scharer, Phys. Plasmas 18, 063501 (2011).

${ }^{34}$ J. L. Domenech-Garret, S. P. Tierno, and L. Conde, J. Appl. Phys. 118, 074904 (2015).

${ }^{35}$ L. Conde, L. F. Ibáñez, and C. Ferro-Fontán, Phys. Rev. E 64, 046402 (2001).

${ }^{36}$ A. V. Phelps, J. Phys. Chem. Ref. Data 20, 557 (1991). 